\title{
Prominent Signet Ring Cell Morphology in a Pulmonary Squamous Cell Carcinoma
}

\author{
Nuri YíĞiT'1 , Ertuğrul ÇELIK'², İbrahim YAVAN ${ }^{1}$ \\ Department of Pathology, 'Gulhane Military Medical Academy and School of Medicine, ANKARA, TURKEY, ${ }^{2}$ Izmir Military Hospital, IZMIR, TURKEY
}

\begin{abstract}
In daily practice, signet ring cell morphology immediately brings to the mind the possibility of an adenocarcinoma at first glance. The signet ring cell appearance has been described and is well-known in a wide variety of some other neoplasms as well. Surprisingly however, neoplastic cells having the same morphology can unexpectedly be encountered in not previously well-documented tumors. Here, we present an 85-year-old man diagnosed with primary pulmonary squamous cell carcinoma and a large signet ring cell population. Examination of the lobectomy specimen and further radiological workup was consistent with stage I disease. Signet ring cell variant of squamous cell carcinoma is a very infrequent tumor and has been reported in only eight cases from skin, cervical and oral cavity biopsies as well as in one case of pulmonary acantholytic variant of squamous cell carcinoma with focal signet ring cells. To be aware of this entity is crucial for pathologists to reach the correct diagnosis, particularly in cytological samples, because of its potentially modifying effect on treatment options and patient management compared to adenocarcinomas. Our patient remained in clinical remission during the 9-month follow-up.
\end{abstract}

Key Words: Signet ring cells, Intracytoplasmic vacuoles, Squamous cell carcinoma, Lung

\section{INTRODUCTION}

The term 'signet ring cell morphology' describes a cell with a large intracytoplasmic vacuole, resulting in compression and eccentric displacement of the nucleus.

Malignant signet ring cells are more commonly seen in the adenocarcinomas, particularly those of gastric, colonic, pancreatic, breast, and lung origin. However, they are not specific to adenocarcinomas and may also rarely be found in a minor subset of various epithelial and non-epithelial neoplasms such as urothelial carcinoma, mesothelioma, leiomyosarcoma, liposarcoma, angiosarcoma, epithelioid sarcoma, gastrointestinal stromal tumor (GIST), and even lymphoma (1-4).

In daily pathology practice, the presence of a dominant population of atypical signet ring cells is almost always associated with a diagnosis of an adenocarcinoma that is specifically called "signet ring cell carcinoma". Although this scenario works in most cases, pathologists should be cautious regarding the risk of overlooking the different types of tumors, although very infrequently encountered, with very similar morphology. Observation of these cells immediately brings to the mind the possibility of an adenocarcinoma at first glance, and this is mostly correct. However, one must be careful during the differential

(Turk Patoloji Derg 2018, 34:194-197)

Received : 08.09.2015 Accepted : 02.10.2015 diagnosis before the final report to be signed out. We would like to remind pathologists that atypical signet ring cells are most frequently seen in adenocarcinomas, but it is very important and crucial to know that they can unexpectedly be seen in other non-adenocarcinoma tumor types that have not been well-described .

Signet ring cell variant of pulmonary squamous cell carcinoma (SCC) is an extremely uncommon neoplasm and only one such case containing focal signet ring cell alteration has been recently described so far. Herein, we present a case of primary pulmonary SCC with a significant signet ring cell morphology.

\section{CASE REPORT}

An 85-year-old man, with a history of dementia, was diagnosed with coronary atherosclerosis and pneumonia. Chest computed tomography (CT) revealed a large mass with an irregular margin in the middle lobe of the right lung. Positron emission tomography-computed tomography (PET-CT) scan did not show any other involvement. The patient underwent concurrent coronary bypass and lobectomy surgery. Grossly, cut surfaces of the middle lobe had an ill-defined, yellowish colored, solid and soft mass, measuring $4.2 \times 3.5 \times 3 \mathrm{~cm}$, around the lobar bronchus.

Correspondence: Nuri YİĞİT

Gulhane Military Medical Academy and School of Medicine,

Department of Pathology, ANKARA, TURKEY

E-mail: nyigitsum@gmail.com Phone: +90 3123043734 
The resection material was fixed in formalin, processed routinely, embedded in paraffin, and stained with hematoxylin and eosin. Histopathological examination showed an infiltration of atypical squamous cells containing optically dense eosinophilic cytoplasm with moderate keratinisation, atypical pleomorphic nuclei, and prominent nucleoli. A few scattered squamous pearls and clearly identifiable intercellular bridges among tumor cells, suggesting squamous origin, were also noted. Interestingly, as an unexpected feature for SCC of the lung, approximately $50 \%$ of the malignant cells contained a single, large and empty intracytoplasmic vacuole which displaced the nucleus peripherally resulting in signet ring cell morphology (Figure 1A-D). Their histologic appearance did not seem to conform with well-known mucin containing carcinoma cells or clear cell histologic variant of SCC. There was no pleural, lymphovascular or perineural invasion and the bronchial surgical margin was free of tumor. Also, the 11 dissected hilar lymph nodes were uninvolved. The remaining lung parenchyma demonstrated focal emphysematous areas, alveolar microlithiasis and anthracotic pigment deposition. The patient had no history or physical examination finding of any extrapulmonary SCC, which might metastasize to the lung. Furthermore, focal in situ carcinoma was observed in the sections and this was convincing evidence for a primary lung tumor.

On immunohistochemical study, the tumor cells were diffusely stained with CK5/6 and p63, but not with TTF-1, CK7, CK20, Napsin A or MOC31 (Figure 1E,F). Based on the findings, a diagnosis of moderately-differentiated signet ring cell variant of pulmonary SCC was made.

Subsequently, relevant histochemical stainings including Periodic Acid-Schiff, Alcian Blue, Mucicarmine as well as Oil Red-O for formalin-fixed and routinely processed tissues were performed to determine the nature of the vacuoles and the results were found to be unremarkable. Furthermore, Adipophilin (clone AP-125) immunostaining was utilized. Whereas both external and internal positive controls (sebaceous glands of the skin appendages and peribronchial innocent adipocytes, respectively) were highlighted with Adipophilin antibody, the vacuoles were negative. We were sure that this appearance was not the result of an artificial effect that can be caused by fixation or the tissue processing procedure because all of the remaining conventional neoplastic cells as well as non-neoplastic host cells were seen with an expected morphology.

The patient was considered to have stage I disease and continued regular follow-up without any additional therapy. The most recent CT scan 6 months after the surgery showed no evidence of recurrence and the patient has been well at the 9-month follow-up.

\section{DISCUSSION}

Depending on the biopsy site and tumor types, the vacuoles of signet ring cells were found to contain large amounts of mucin, glycogen, lipid, or immunoglobulin $(5,6)$. Additionally, artificial or degenerative changes can cause such morphology. Most of the accumulated substances can easily be identified by specific histochemical or immunohistochemical auxiliary studies such as anti-MUC antibodies and Mucicarmine stain for mucin, Periodic Acid-Schiff stain for glycogen, and Oil Red-O stain for lipids, particularly in fresh tissues. Artificial or degenerative changes may also be disclosed with an intimate comparison of the neighboring cellular components.

In addition to classical SCC, different histologic variants such as basaloid, clear cell, acantholytic, spindle, pigmented, papillary, non-keratinizing, and pleomorphic are welldescribed $(7,8)$. Signet ring SCC was first reported in a skin tumor by Cramer and Heggeness (9). Only eight cases of extrapulmonary signet ring SCCs, including 5 cutaneous SCC, 2 cervical SCC and one oral cavity SCC, have been documented until now (9-15). To the best of our knowledge, there is only one case report that has been recently published about an acantholytic variant of pulmonary SCC with focal signet ring cell morphology (16).

Although the appearance of the vacuoles is reminiscent of the macrovesicular lipid droplet, their nature could not be exactly elucidated in the previously described signet ring SCCs, as was in our case. Kupryjanczyk and Kujawa described two cases of cervical SCC with signet ring cell histology where electron microscopy indicated multiple oval or irregular empty spaces within the cytoplasm, but their nature could not be determined (10). McKinley et al. reported a signet ring cell SCC of the skin (11). They performed ultrastructural examination and found that the vacuoles were large dilatations of cisternae of the rough endoplasmic reticulum. We did not encounter any other explanatory data to clarify their origin in SCCs, in the literature review. Although not specific for all types of lipid molecules, we studied Adipophilin immunohistochemistry and other histochemical stainings for mucinous accumulations, and concluded that these intracytoplasmic vacuoles were neither likely be caused by lipid or mucin based on the negative stainings.

Differential diagnosis mainly includes adenocarcinoma with or without signet ring cells. To be aware of that pulmonary 

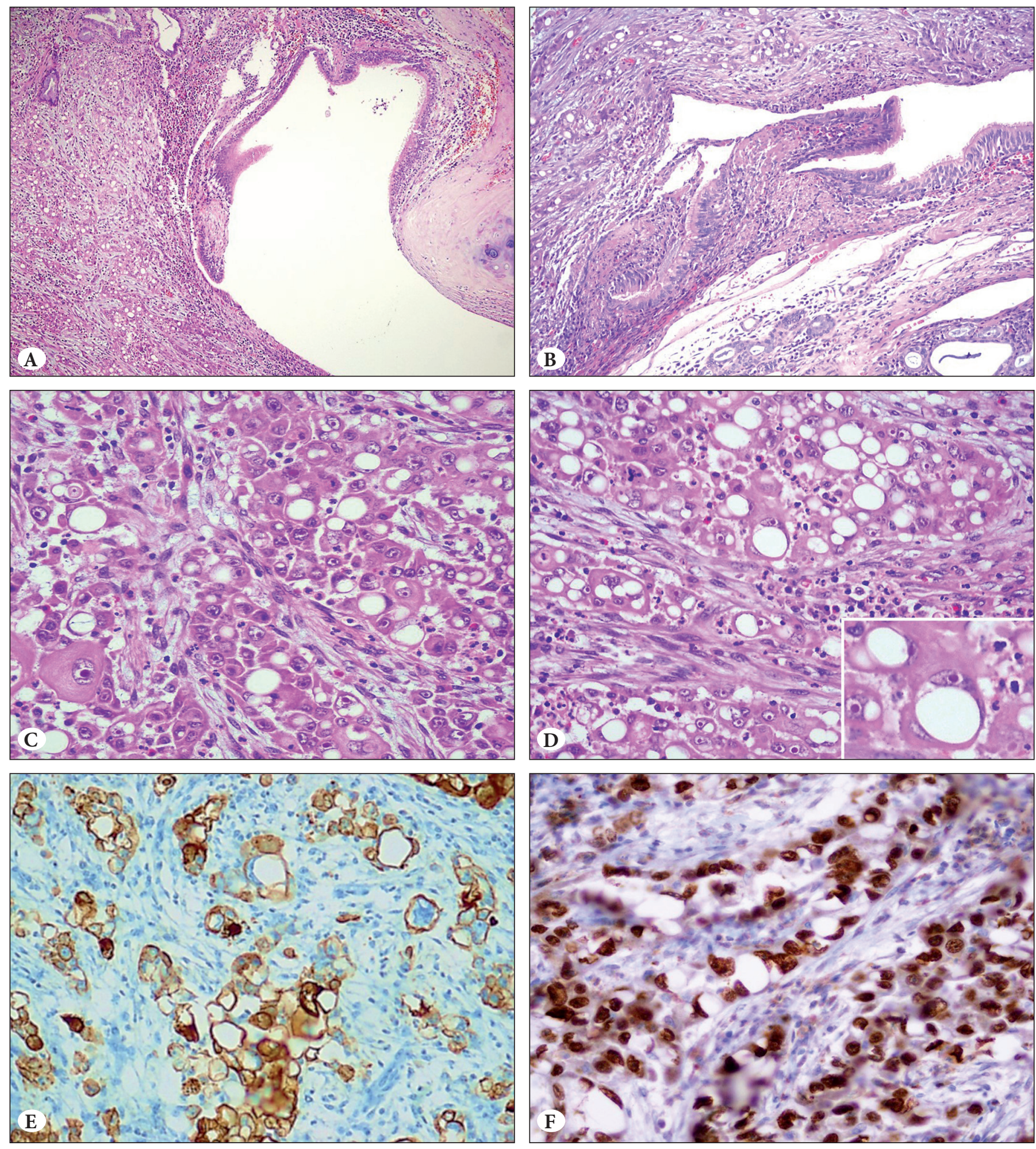

Figure 1: A,B) Pulmonary squamous cell carcinoma surrounding lobar bronchus (H\&E; x100). C,D) Many tumor cells contain a single, large and clear intracytoplasmic vacuole pushing nuclei to the periphery and causing an obvious signet ring cell appearance (inset) (H\&E; $\mathrm{x} 400$ ). E) CK5/6 antibody sparing the vacuoles and highlighting the concentric ring of cytoplasm of the neoplastic cells (CK5/6; 200 ). F) Strong p63 expression in the tumor cells (p63; x400). 
SCC can exhibit signet ring cell morphology and the distinction between signet ring SCC and adenocarcinoma is crucial to render the correct diagnosis because of the different patient management. In particular, this issue can be very challenging in cytological samples owing to the possible inadequate specimens for necessary ancillary techniques such as immunocytochemistry. Histopathologically, a dense eosinophilic cytoplasm, pleomorphic atypical nuclei, existence of keratinisation, dyskeratotic cells, whorled pattern, squamous pearls, and intercellular bridges provide definite evidence of squamous differentiation of the tumor and reliable clues for discriminating from an adenocarcinoma in the differential. The oresence of an in situ component in the sections and absence of preexisting or synchronous SCC in different locations are strong supportive features to exclude the possibility of a metastasis from extrapulmonary sites. Additionally, positive staining with CK5/6, p63 and p40 while lack of any immunoreactivity against TTF-1, CK7, Napsin-A, and MOC-31 favors the diagnosis of SCC.

Due to its rarity, it is not clear whether the pulmonary signet ring variant of SCC has a different clinical course or therapy response depending on the stage compared to conventional cases. Nine months after the surgery, our patient has been doing well without any signs of recurrence. Nevertheless, the biological behavior needs to be further analyzed to determine the most effective clinical management.

In conclusion, the signet ring variant of SCC should be kept in mind in addition to the commonly suspected adenocarcinomas on examination of lung biopsies consistent with a malignancy showing signet ring cell morphology,. Specific morphological features and immunoexpression profile of SCC as well as the presence of an in situ component help to make the correct diagnosis. To rule out a metastasis, the possibility of a non-pulmonary SCC should be queried at the time of biopsy or in the patient's history before making a definite diagnosis.

\section{ACKNOWLEDGEMENT}

We would like to thank Dr. Şule Öztürk Sarı from Istanbul University Capa Faculty of Medicine, for helping us with some confirmatory immunohistochemical studies.

\section{REFERENCES}

1. Terada T. Primary pure signet-ring cell adenocarcinoma of the urinary bladder: A report of three cases with an immunohistochemical study. Med Oncol. 2012;29:2866-9.

2. Karabagli P, Kilic H. Primary pure signet cell carcinoma of the breast: A case report and review of the literature. Breast Cancer. 2013;20:363-6.

3. Salviato T, Bacchi CE, Luzar B, Falconieri G. Signet ring cell angiosarcoma: A hitherto unreported pitfall in the diagnosis of epithelioid cutaneous malignancies. Am J Dermatopathol. 2013;35:671-5

4. Bogusz AM, Tierno B, Brown D, Pihan G. Extreme signet ring cell change in a large B-cell lymphoma of follicular origin. Int J Surg Pathol. 2013;21:399-403.

5. Grier DD, Robbins K. Signet-ring plasma cell myeloma. Am J Hematol. 2012;87:625.

6. Iwasaki H, Ishiguro M, Nishio J, Aoki M, Yokoyama R, Yokoyama K, Taguchi K, Nabeshima K. Extensive lipoma-like changes of myxoid liposarcoma: Morphologic, immunohistochemical, and molecular cytogenetic analyses. Virchows Arch. 2015;466:453-64.

7. Saito T, Mitomi H, Yao T. Molecular pathology and potential therapeutic targets in esophageal basaloid squamous cell carcinoma. Int J Clin Exp Pathol. 2015;8:2267-73.

8. Mehrad M, Carpenter DH, Chernock RD, Wang H, Ma XJ, Luo Y, Luo J, Lewis JS Jr, El-Mofty SK. Papillary squamous cell carcinoma of the head and neck: Clinicopathologic and molecular features with special reference to human papillomavirus. Am J Surg Pathol. 2013;37:1349-56.

9. Cramer SF, Heggeness LM. Signet-ring squamous cell carcinoma. Am J Clin Pathol. 1989;91:488-91.

10. Kupryjanczyk J, Kujawa M. Signet-ring cells in squamous cell carcinoma of the cervix and in non-neoplastic ectocervical epithelium. Int J Gynecol Cancer. 1992;2:152-6.

11. McKinley E, Valles R, Bang R, Bocklage T. Signet-ring squamous cell carcinoma: A case report. J Cutan Pathol. 1998;25:176-81.

12. El Demellawy, Onuma K, Alowami S. Signet ring squamous cell carcinoma-the forgotten variant: Case report and review of the literature. J Cutan Pathol. 2011;38:306-8.

13. Lortscher DN, Satter EK, Romero LS. Signet ring-like cells: No longer a "signature" of glandular differentiation. Dermatol Online J. 2012;18:3.

14. Nakajima K, Kaneko T, Aizu T, Nakano H, Matsuzaki Y, Sawamura D. Signet-ring cutaneous squamous cell carcinoma arising on the back of the finger. Case Rep Dermatol. 2013;5:215-8.

15. Alves MO, de Sousa S, Carvalho Y, Almeida J. Primary signetring squamous cell carcinoma of the oral cavity: The first intraoral case. Head Neck Oncol. 2014;6:11.

16. Park HS. Acantholytic squamous cell carcinoma showing significant signet ring cell component of the lung. Histopathology. 2015 Jun 25. [Epub ahead of print] 